\title{
Impact of Myalgic Encephalomyelitis on treatment of comorbidities: A lived experience
}

\author{
Denise Lopez-Majano \\ Chester Springs, PA, USA \\ E-mail: speakupaboutme@gmail.com
}

Received 12 September 2019

Accepted 10 December 2019

\begin{abstract}
Myalgic Encephalomyelitis (ME) is a complex, chronic, disabling, multi-system disease with no FDA-approved treatments. ME greatly impacts quality of life $(\mathrm{QoL})$ with studies showing that people with ME often have worse quality of life than people with sickle cell anemia and cystic fibrosis, among other chronic diseases. People with ME frequently have comorbidities, which, if treated, could improve quality of life. However, the pervasive impact of ME makes treatment of comorbidities difficult. When trying to treat comorbidities it is therefore important for rehabilitation specialists to understand the impact of ME on day-to-day life in order to avoid treatment-related harms or exacerbation of ME symptoms. This article details the lived experience of one family in which both siblings have ME and comorbidities.
\end{abstract}

Keywords: Myalgic Encephalomyelitis, postural orthostatic tachycardia syndrome, joint hypermobility syndrome, comorbidities, management

\section{Introduction}

My two children were healthy, active, and engaged with family and friends and the world around them. They were competitive swimmers and good students. Among other things, they enjoyed being goofy and kind, learning, having fun, and traveling. In other words, they were active participants in life.

That all changed when one of my sons, suddenly at age 12 (in 2005) and then my other son, gradually beginning at age 15 (2006), developed myalgic encephalomyelitis (ME). Now they are housebound, partially bedbound, and need around-the-clock care.

Since the onset of ME my sons have constant incapacitating symptoms including unrelenting reduction in cognitive and physical function, fatigue and pathological exhaustion, orthostatic intolerance, cognitive impairment, unrefreshing sleep and altered sleep schedules, migratory pains, syncopal and pre-syncopal episodes several times a week, extended episodes of body temperature dysregulation, always feeling sick, or as though they have an infection, heightened sensitivity to light, sound, smell, and touch, and extreme sensitivity to changes in air quality (despite central air conditioning, filters, etc). Cognitive impairment is evidenced by marked reduction in response time, working memory, inability to converse for more than three to five minutes at a time, inability to multi-task, and markedly reduced ability to learn new things at a typical pace. Going beyond their very reduced cognitive and physical resources results in post-exertional malaise (aka PEM, postexertional neuroimmune exhaustion (PENE), crash, collapse) lasting for weeks during which their cognitive and physical function are further reduced and other symptoms are exacerbated also.

Following the onset of ME, we learned that my sons, like many people with ME, have several 
comorbidities [1] including orthostatic intolerance (OI) [2], JHS (Ehlers-Danlos syndrome hypermobility type) [3], cervical spine instability and Raynaud's disease. Successful treatment of my sons' comorbidities could improve their quality of life, but because of ME, my sons cannot manage the necessary standard treatment without significant complications and/or setbacks. In people who have OI or EDS but who do not have ME, there are several treatments that can be effective. However, my sons have ME which makes treatment of comorbidities more challenging.

For instance, people with POTS (postural orthostatic tachycardia syndrome, a form of OI) and/or JHS are usually referred for physical and/or occupational therapy. Typically, therapy involves appointments at least twice a week for several months with a focus on building strength and endurance to help improve quality of life. But even if my sons did their therapy at home to avoid the exertion involved in leaving the house, twice weekly is too frequent to allow them to recover to their baseline level of function between appointments. My sons and most people with ME cannot tolerate the usual duration and intensity of therapy appointments for POTS, JHS, etc. People with ME often cannot build endurance. Their quality of life is lower than that of people with cystic fibrosis, epilepsy, sickle cell anemia, and type 1 diabetes (among others) [4]. ME complicates the treatment of comorbidities because of the often unpredictable limitations it imposes on cognitive and physical exertion. I define exertion as any physical or cognitive effort.

\section{What is Myalgic Encephalomyelitis (ME)?}

ME is a complex, chronic, multi-system disease that affects all ages, genders, ethnicities, socioeconomic groups, and educational levels. Estimates indicate there are over 800,000 people with ME in the United States [1,9] and millions more worldwide.

At a minimum, people with ME contend with an unrelenting reduction in cognitive and physical resources, fatigue that is not alleviated by rest, unrefreshing sleep, and orthostatic intolerance and/or cognitive impairments - none of which pre-date disease onset. Symptoms of ME must be present at least 50 percent of the time, be of at least moderate intensity and have persisted for at least 6 months. Often people with ME have significantly heightened sensitivity to light, sound, odors, and touch. Many are also unusually sensitive to small amounts of medications - hence the advice with all treatment, to start low and go slow. As with many chronic diseases, ME has a range of severity. Some people with ME can care for themselves and/or live independently. Many others are housebound or bed bound and up to 75 percent of people with ME cannot work [5]. Some people with $\mathrm{ME}$ are so very severely ill they are completely bed bound and must be tube-fed [6]. People with ME are inordinately reactive to cognitive and/or physical exertion which makes them very vulnerable to postexertional malaise (PEM, aka crash/collapse/PENE) [7-9].

This hallmark (PEM) of ME is a profound worsening of exhaustion and many of the other symptoms that the individual usually experiences. PEM further reduces a person's level of cognitive/physical function for days, weeks, even months. PEM can be triggered by minimal cognitive or physical exertion. And when I say minimal, I mean that for some people with ME, things like brushing one's teeth or having even a brief conversation can trigger PEM. Sometimes, PEM can set in without a discernible trigger.

Studies indicate that PEM involves impaired aerobic energy metabolism and impaired oxygen consumption [10]. The 2-day CPET (cardio-pulmonary exercise testing) tests show that people with ME switch from aerobic to anaerobic metabolism far earlier than healthy people [11]. The result is that ME significantly reduces people's level of physical and cognitive function as compared to their pre-illness levels and as compared to healthy individuals.

Biomedical research for ME has been woefully underfunded for decades. One consequence of this lack of funding is that there is little information on the natural history (progression) of ME. There are very few specialists in ME and it is rarely taught in medical schools. There are no FDA-approved treatments, so at best, people with ME get treatments that attempt to ameliorate symptoms. Over the years my sons have tried every treatment recommended by the specialist we are fortunate to be able to see - a rarity in and of itself as most people with ME cannot get an appointment with a specialist, are on long waiting lists if they do, and often cannot make it out of their home to see the specialist regardless. But attempted treatments have not resulted in much improvement in my sons' level of function and quality of life.

\section{Impact of ME on my sons}

My sons are severely disabled because of ME. They are housebound and unable to care for them- 
selves. They were so young when they were stricken with ME and now are so disabled by ME that they haven't been able to develop the skills needed to live independently. Because of the pervasive impact of ME, my sons have also not been able to complete their formal education.

In 2020, the best that people with ME can hope for is to be at their baseline level of cognitive and physical function. But baseline is a moving target it is unpredictable.

My sons are very limited in what they can do and for how long. They each have different limits to their cognitive and physical resources. There are few activities of daily living they can do consistently. Attempting to do more would overtax their limited cognitive and physical resources and leave them even more vulnerable to PEM.

\section{Current daily level of function}

The best my sons can currently hope for in physical day-to-day function is as follows:

- have a brief shower twice a week using a shower chair

- go up and down the stairs 3-4 times a day (spaced out over the course of the day)

- leave the house - perhaps once every 6 weeks for one son, perhaps once every 3 months for the other

- walk a few hundred steps a day

- clear their place at the table (though often they cannot load or unload the dishwasher because stooping/bending over/crouching triggers tachycardia)

- engage in quiet activity on their computers for an hour at a time (followed by at least the same amount of rest time).

ME does not impair intelligence, but it significantly impacts cognitive function in many people with ME. The best my sons can currently hope for in cognitive day-to-day function is as follows:

- learn new material - max 20 minutes, two times a week

- listen to music - about 30 minutes at a time, perhaps three times a week

- read familiar books - maybe an hour and a half, two times a week

- read new books - about 30 minutes, two times a week
- decide what they will drink with their meal (which I make)

- generate a bit of original thought - for instance request a particular food

- engage in a short conversation (three to five minutes max) - but without much noticeable emotion as that is too taxing.

Over the years we've come to rely on non-verbal communication. A shrug, a tilt of the head or a hand gesture is often all they can manage. Eye contact is acknowledgment. A raised eyebrow, an upturned corner of the mouth, a slight nod of the head - for my sons, those are big reactions. Often I can recognize things that may not be readily apparent to others change in skin color, glazed or bleary eyes, "zoned out", unresponsiveness, etc. and can assess what these things mean in terms of my sons' level of function at that point in time. With me, my sons don't have to explain as much because throughout their illness I have been their caregiver and am all too aware of the impact of ME, the impact of exertion, and the impact of comorbidities.

Most healthcare providers we have met have never encountered people with ME who are as severely disabled as my sons are. This is largely because most people with ME have great difficulty accessing health care and getting to appointments. There are very few practitioners who are truly knowledgeable about $\mathrm{ME}$ and its repercussions. Even the most common comorbidities are not familiar to every healthcare provider. To add to the complexity, the comorbid conditions often have subtypes as well. For example, orthostatic intolerance has several subtypes, including Postural Tachycardia Syndrome (POTS) and Neurally Mediated Hypotension (NMH). Ehlers-Danlos Syndromes (EDS) are a group of more than a dozen types. People with ME and their healthcare providers face the challenge of sifting through the symptoms of ME to uncover comorbidities, then filtering and sifting further to identify the subtype of a comorbidity, and only then beginning the often lengthy search for effective treatment.

\section{Impact of ME and comorbidities on my sons}

Our experience with just one of my sons' comorbid conditions is illustrative. Their doctor suspects the comorbidity is present, but accurately determining the subtype requires at least two types of specialized 
imaging and an appointment with another specialist to discuss treatment options. Depending on the subset, treatment for this comorbidity might involve physical therapy, injections or surgery.

To get the necessary imaging we must drive several hours each way. Traveling for healthcare is common for people with ME because of the dearth of knowledgeable providers, yet traveling has a huge negative impact on my sons. Anywhere my sons go, I must take them. They've never been well enough to learn to drive. During appointments it is often simpler if I speak for them, as it takes so much out of them to even be at appointments. Talking exhausts them (physically and cognitively) even more. Many people with ME don't have the advantages of a parent and/or caregiver, and those even more severely ill than my sons cannot leave the house at all and as a result often have to go without health care.

Before we go anywhere my sons must rest preemptively, ideally for at least two weeks. During this time their level of exertion is further reduced below the day-to-day physical and cognitive functioning described above. After any event (appointments, seeing friends, etc) it takes several weeks of continued reduced exertion for PEM to resolve and for them to return to their baselines. Spontaneity is not an option for us. If one must reduce already very limited activity levels for two weeks or more before and after every event, opportunities for events of any kind are much reduced compared to levels of healthy people.

In preparation for travel I must determine the route, travel time (including a buffer for weather, traffic, and some "quiet" time before appointments), necessary expenditures (tolls, parking, gas, food, etc), parking options, and distance from parking to appointment (does it warrant wheelchairs?). I make sure we have a full tank of gas, pillows (for under and around their bodies), eye covers, ear plugs, cervical collars (a precautionary measure because of joint hypermobility syndrome and cervical spine instability), blankets, sunglasses, snacks, drinks, barf bags and wipes, medications, notes and updated medication lists for healthcare professionals, as well as everything necessary to quickly prepare a meal when we get home, including additional meal options to accommodate what they each can tolerate at that time.

We endeavor to do as much as possible to minimize vulnerability to PEM. All the while I am cognizant that despite all our efforts, adding in any activity will likely result in weeks-long payback (the effects of the crash/PEM/etc) stemming from having exceeded their limits.
My sons would very much like to once again be productive individuals and lead full lives. Therefore, we are determined to address as many comorbidities as possible within the parameters imposed by ME because every improvement in my sons' quality of life is worth it.

\section{Recommendations based on our experiences}

Because ME impacts every moment of the lives of people with ME, rehabilitation specialists (physical therapists, occupational therapists, etc.) must do the following in order to help people with ME:

- understand PEM, hypersensitivities, reduced cognitive and physical resources, unrefreshing sleep, and pervasive exhaustion

- streamline therapy for people with ME

- reduce frequency/appointment duration/intensity of treatment but also expect treatment to take much longer than usual

- be mindful that if the person with ME does not have a caregiver to help convey things, progress may be even slower due to the dual effort of communication and therapy

- anticipate periods of time during which people with ME cannot attend appointments because of physical and cognitive limitations

- ensure people with ME are not penalized for missed appointments

- know that because of limited cognitive and physical resources it may be necessary to repeat exercises/activities and rephrase instructions to ensure they are accurately learned

- keep in mind that each person with ME has different limits and these may also vary from one moment/hour/day to the next

- know that when it comes to exertion, the person with ME must be the one to determine each time if exertion is in any way feasible

- understand that assessment and treatment of comorbidities in people with ME requires flexibility on the part of rehabilitation specialists.

\section{Conclusion}

To ensure the greatest chance of successful treatment of comorbidities in people with ME, rehabilitation specialists must be cognizant of the impact of ME 
on cognitive and physical function and must individualize treatment modalities in order to accommodate persons with ME. Careful adaptation of treatment modalities will increase the possibility of successful treatment of comorbidities in people with ME.

\section{Acknowledgments}

Thank you to Wilhelmina Jenkins, Jennifer Spotila and Dr. Peter Rowe for their review of this article.

\section{Conflict of interest}

None to report.

\section{Helpful resources}

Chronic Fatigue Syndrome Myalgic Encephalomyelitis, Primer for Clinical Practitioners: 2014 Edition

Myalgic Encephalomyelitis/Chronic Fatigue Syndrome Diagnosis and Management in Young People: A Primer

Beyond Myalgic Encephalomyelitis/Chronic Fatigue Syndrome: Redefining an Illness (2015)

Beyond Myalgic Encephalomyelitis/Chronic Fatigue Syndrome: Redefining an Illness: Report Guide for Clinicians

\section{References}

[1] New York State Department of Health, Myalgic Encephalomyelitis ("Chronic Fatigue Syndrome") webpage, section How is ME Diagnosed? [Revised: May 2018; cited 8 Sept 2019] Available from: https://www.health. ny.gov/diseases/conditions/me-cfs/

[2] Rowe PC. General information brochure on orthostatic intolerance and its treatment. Chronic Fatigue Clinic, Johns Hopkins Children's Center [March 2014; cited 8 Sept 2019] Available from: https://www.dysautonomiainternational. org/pdf/RoweOIsummary.pdf

[3] Tinkle B, Castori M, Berglund B, Cohen H, Grahme R, Kazkaz H, Levy H. Hypermobile Ehlers-Danlos syndrome (a.k.a. Ehlers-Danlos syndrome Type III and Ehlers-Danlos syndrome hypermobility type): Clinical description and natural history. Volume175, Issue1 Special Issue: The Ehlers-Danlos Syndromes: Reports from the International Consortium on the Ehlers-Danlos Syndromes. 2017;48-69.

[4] Roma M, Marden CL, Flaherty MAK, Jasion SE, Cranston EM and Rowe PC. Impaired Health-Related Quality of Life in Adolescent Myalgic Encephalomyelitis/Chronic Fatigue Syndrome: The Impact of Core Symptoms. Frontiers in Pediatrics. 2019

[5] The Norwegian ME Association. Bringsli GJ, Gilje AM, Getz Wold BK. The Norwegian ME Association National Survey: Abridged English version. Oslo, Norway: 2014. [published 2014; cited 8 Sept 2019] Available from: http://www.me-foreningen.info/wp-content/uploads/ 2016/09/ME-Nat-Norwegian-Survey-Abr-Eng-Ver.pdf

[6] Prior R, CNN. He pioneered technology that fueled the Human Genome Project. Now his greatest challenge is curing his own son [Updated 11:02 AM ET, Mon May 13, 2019; cited 8 Sept 2019] Available from: https://www.cnn. com/2019/05/12/health/stanford-geneticist-chronic-fatigue -syndrome-trnd/index.html

[7] Carruthers BM, Jain AK, De Meirleir KL, Peterson DL, Klimas NG, Lerner AM, Bested AC, Flor-Henry P, Joshi P, Powles ACP, Sherkey JA, van de Sande MI. Myalgic Encephalomyelitis/Chronic Fatigue Syndrome: Clinical Working Case Definition, Diagnostic and Treatment Protocols (PDF), Journal of Chronic Fatigue Syndrome. 2003;11(2):7-115.

[8] Carruthers BM, van de Sande MI, De Meirleir KL, Klimas NG, Broderick G, Mitchell T, Staines D, Powles AC, Speight N, Vallings R, Bateman L, Baumgarten-Austrheim B, Bell DS, Carlo-Stella N, Chia J, Darragh A, Jo D, Lewis D, Light AR, Marshall-Gradisnik S, Mena I, Mikovits JA, Miwa K, Murovska M, Pall ML, Stevens S. Myalgic encephalomyelitis: International Consensus Criteria. J Intern Med. 2011;270(4):327-338.

[9] Beyond Myalgic Encephalomyelitis/Chronic Fatigue Syndrome: Redefining an Illness. Committee on the Diagnostic Criteria for Myalgic Encephalomyelitis/Chronic Fatigue Syndrome; Board on the Health of Select Populations; Institute of Medicine. Washington (DC): National Academies Press (US). 2015.

[10] CDC. Myalgic Encephalomyelitis. (webpage) Etiology and Pathophysiology. Section Cellular metabolism abnormalities. [Page last reviewed: July 12, 2018; cited 8 Sept. 2019] Available from https://www.cdc.gov/me-cfs/healthcareproviders/presentation-clinical-course/etiology-pathophysiology.html

[11] Keller BA, Pryor JL, Giloteaux L. Inability of myalgic encephalomyelitis/chronic fatigue syndrome patients to reproduce $\mathrm{VO}_{2}$ peak indicates functional impairment. Journal of Translational Medicine. 2014;12:104. 